\title{
Optical Modeling Analysis of Red, Green, and Yellow Phosphors with a Blue LED
}

\author{
Wen-Shing Sun ${ }^{1}$ and Chuen-Lin Tien (D) $^{2}$ \\ ${ }^{1}$ Department of Optics and Photonics, National Central University, Chungli 32001, Taiwan \\ ${ }^{2}$ Department of Electrical Engineering, Feng Chia University, Taichung 40724, Taiwan
}

Correspondence should be addressed to Chuen-Lin Tien; cltien@fcu.edu.tw

Received 21 January 2018; Accepted 12 March 2018; Published 1 August 2018

Academic Editor: Shuan-Yu Huang

Copyright (C) 2018 Wen-Shing Sun and Chuen-Lin Tien. This is an open access article distributed under the Creative Commons Attribution License, which permits unrestricted use, distribution, and reproduction in any medium, provided the original work is properly cited.

\begin{abstract}
The luminous properties of red, green, and yellow phosphors with a blue LED are evaluated and a corresponding optical model is constructed using the optical simulation software LightTools ${ }^{\circledR}$. According to the phosphor particle model, the desired chromaticity coordinate of the multicomponent light which is excited by a blue LED can be achieved by modifying the weight concentration of the phosphors. A comparison of the four types of LED package modules tested shows that the yellow phosphor encapsulated with a semispherical module takes up a lesser percentage of the total weight percentage of a constructed white light with a correlated color temperature of around $4000 \mathrm{~K}$. The simulation and experimental results provide a cross-reference for better packaging and encapsulating designs for lumen improvement.
\end{abstract}

\section{Introduction}

The white light LED has become a popular choice to replace traditional lighting sources. Compared to incandescent and fluorescent lamps, white LEDs have many advantages, such as a long lifetime, high efficiency, and lower energy consumption [1]. Most white LEDs use phosphors excited by diode chips with short wavelengths (blue or violet) to reemit a broad spectrum light with a good color-rendering index [24]. Since the optical performance of LED lighting, including the correlated color temperature (CCT) values, is strongly dependent on the thickness, combination concentration, and geometrical distribution of the phosphors, the commercially available recipes for white LEDs usually require careful manipulation if they are to meet the CCT requirements. Any modification to the pattern of the package could change the volume, density, concentration, or uniformity of the luminescent phosphor film and cause variations in the CCT, chromaticity coordinates, and luminous efficacy.

To empirically determine the factors that dominate the optical properties of the white light LED, detailed experiments must be carried out. In terms of time savings and cost effectiveness, a proper optical model of phosphors is most beneficial in reducing the trial-and-error process. In order to establish an LED model, experiments and simulations dealing with the relationships between the encapsulants, package structures, performance of the radiation power spectrum, CCT, and chromaticity coordinates must be conducted and the results analyzed. Further, some special LED package designs with higher uniformity but using lesser phosphor can be considered as an adjunct of the proposed model [5]. Yang et al. [6] demonstrated the accuracy feedback method of chromaticity coordinates and spectra. This feedback coating method offers an easy approach towards optimized spectra. Wang and Huang [7] also reported the chromaticity coordinates and spectra of phosphor-converted LEDs are well controlled by the feedback coating method. Our phosphor particle model was designed to simulate the scattering and absorbing process of light inside the phosphors. This model was based on the Mie theory, the ray tracing method, and Monte-Carlo algorithms [8-11]. A blue light LED was used to excite the phosphors, with the samples numbered R-645 and R-626 indicating red lights, G-531 and G-529 indicating green lights, and Y-561 yellow light. The number of the samples indicates the peak value of the emission spectrum in nanometers produced by the phosphor. For example, the 


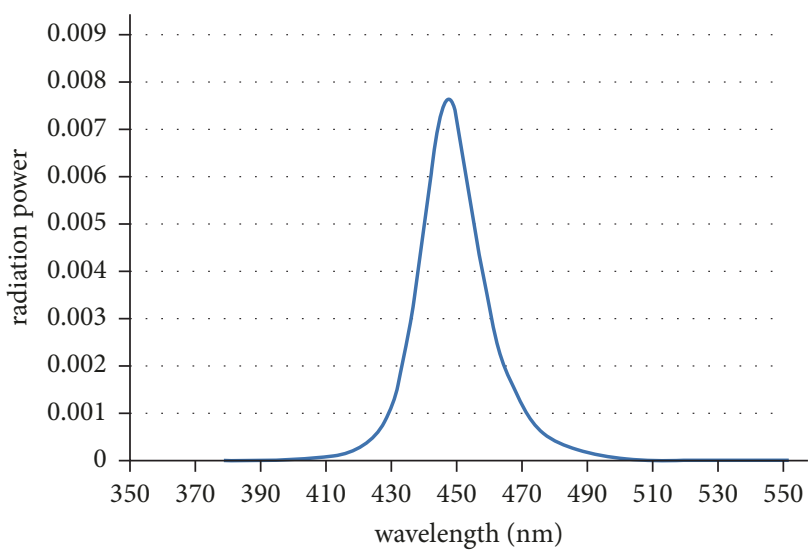

(a)

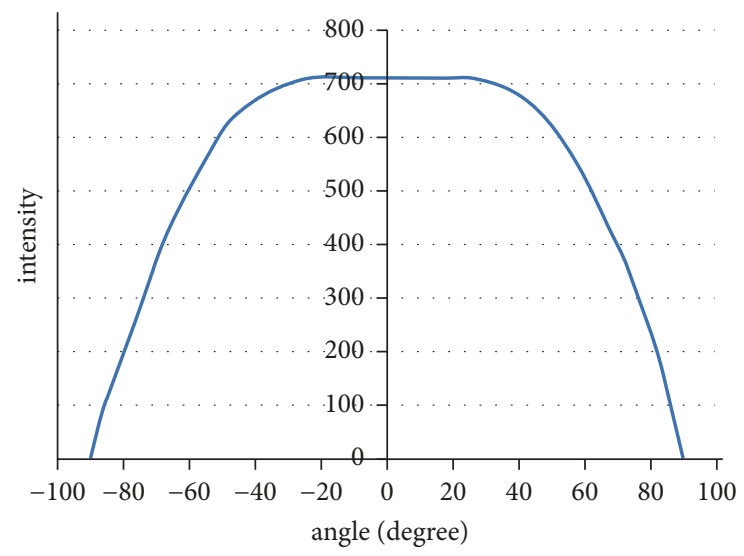

(b)

FIGURE 1: (a) Radiation power spectrum and (b) luminous intensity angular distribution curve of the blue LED light source.

peak value of the R- 645 phosphor is $645 \mathrm{~nm}$. The LED power efficiency at a CCT value of about $4000 \mathrm{~K}$ for the different package types was compared with this phosphor particle model. The simulation and experimental results should provide a reference to improve the luminous properties for better packaging and encapsulating designs.

\section{Principle}

The characteristic parameters of the blue LED, the spectra of the phosphors, and the power transform efficiency of the phosphor powders acting with blue light were established.

2.1. Blue LED Light Source. The dimensions of the blue LED chip were $0.97 \mathrm{~mm} \times 0.51 \mathrm{~mm} \times 0.11 \mathrm{~mm}$. Figure $1(\mathrm{a})$ shows the radiation power distribution versus wavelength with a peak value of $447 \mathrm{~nm}$; Figure 1(b) shows the angular distribution of the luminous intensity curve.

\subsection{Blue LED Source Spectrum, Excitation, and Emission} Spectra of the Phosphors. Figure 2 shows the excitation and emission spectra of the R-645, R-626, G-531, and G-529 phosphors and the spectra of the pumping source for the experimental results. The blue LED spectrum is indicated by the blue line with a peak value of $447 \mathrm{~nm}$, the green lines indicate the excitation spectra of the phosphors, and the red lines show the reemission spectra of phosphors with a longer wavelength shift under the blue LED pumping.

2.3. Spectra Analysis of Different Weight Concentrations of Phosphor under the Blue LED Excitation. The radiation power and chromaticity coordinates of four monochromic phosphors, the red phosphors, R-645 and R626, and the green phosphors, G531 and G529, at different weight percentages were measured. Figure 3 shows the schematic structure of the blue LED with a uniformly distributed phosphor film used in this study. The weight concentrations of the four different phosphors are shown in Table 1.
TABLE 1: List of weight concentrations of the four different phosphors.

\begin{tabular}{lccc}
\hline Type & \multicolumn{3}{c}{ Weight concentration of phosphors } \\
\hline R-645 & $3 \%$ & $5 \%$ & $10 \%$ \\
R-626 & $0.83 \%$ & $1.37 \%$ & $2.11 \%$ \\
G-531 & $5 \%$ & $10 \%$ & $20 \%$ \\
G-529 & $8 \%$ & $11.2 \%$ & $15 \%$ \\
\hline
\end{tabular}

A normalized cross-correlation (NCC) formula was adopted to evaluate the fitness between the simulation and empirical data [12]. The NCC is given by

$$
=\frac{(1 / N) \sum_{\lambda=380}^{\lambda=780}\left(A_{\lambda}-\bar{A}\right)\left(B_{\lambda}-\bar{B}\right)}{\sqrt{(1 / N) \sum_{\lambda=380}^{\lambda=780}\left(A_{\lambda}-\bar{A}\right)^{2}} \sqrt{(1 / N) \sum_{\lambda=380}^{\lambda=780}\left(B_{\lambda}-\bar{B}\right)^{2}}},
$$

where $A_{\lambda}$ and $B_{\lambda}$ are the values of the radiation power at certain wavelengths, $\lambda ; \bar{A}$ and $\bar{B}$ are the values of the average radiation power for the simulations and experiments, respectively; and $N$ is the number of experiments and the number of wavelengths to be evaluated.

\section{Simulation Results}

The empirical and simulated data curves of the radiation power spectra of phosphors of different weight percentages under blue light excitation are discussed in Sections 3.1-3.4.

3.1. Comparison of the Radiation Power Spectra and CIE Chromaticity Locations of Phosphor R-645 with Different Weight Concentrations. Figure 4 shows the radiation power of phosphor R-645 with different weight concentrations (3\%, $5 \%$, and $10 \%$ ) excited by a blue LED. The blue line indicates 


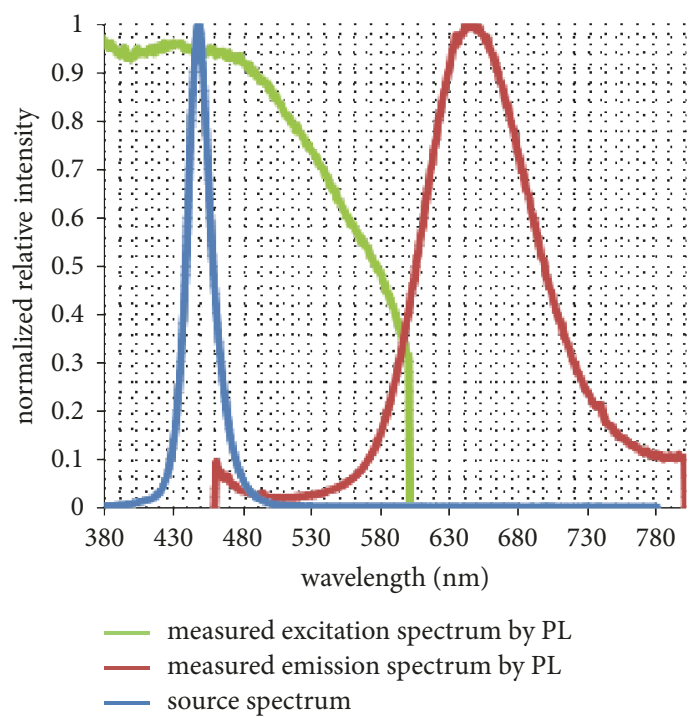

(a)

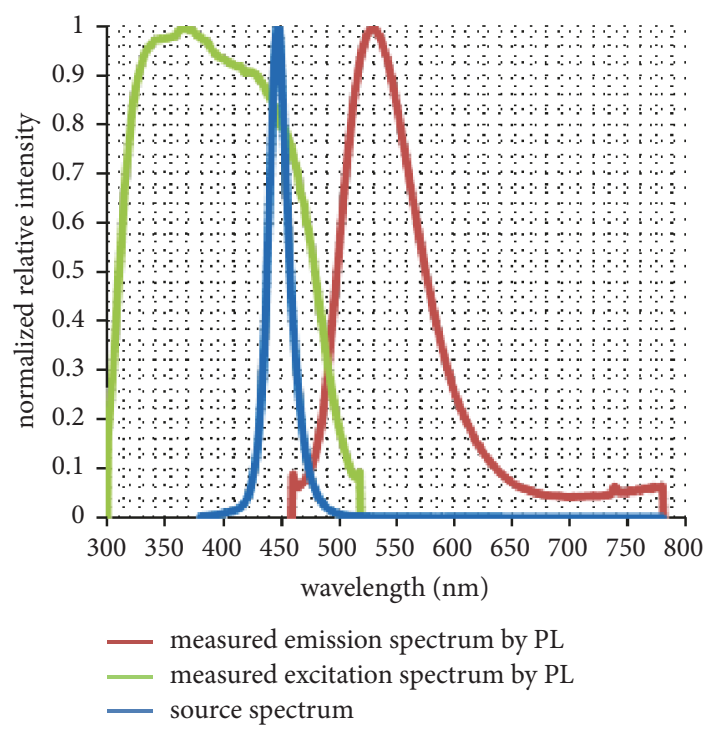

(c)

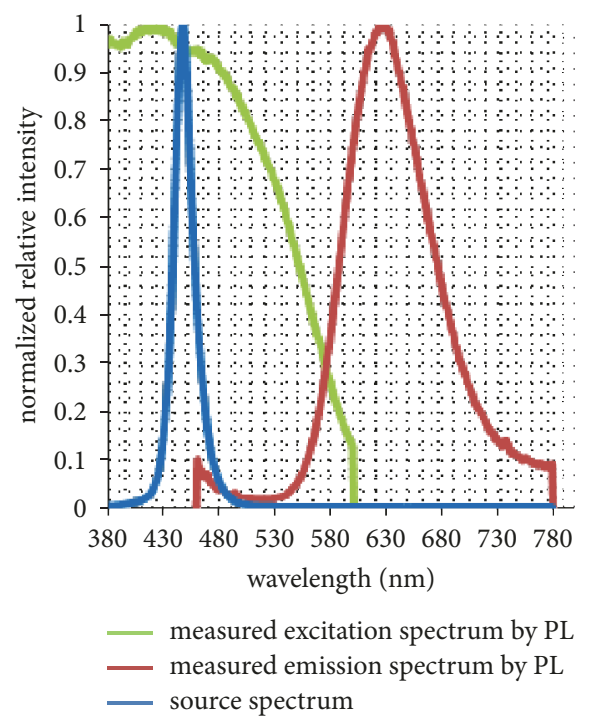

(b)

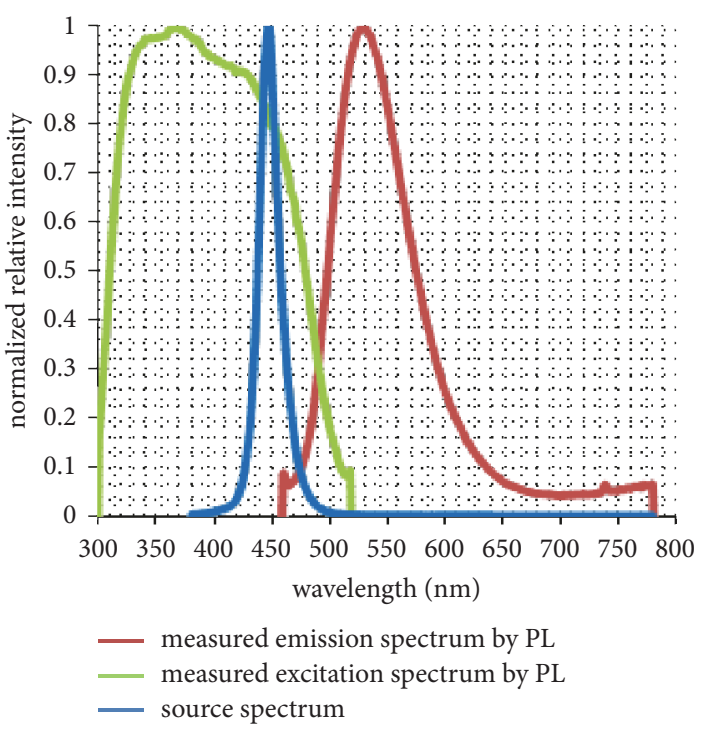

(d)

Figure 2: Empirical emission spectrum of the blue LED and the excitation and emission spectra corresponding to phosphors (a) R-645, (b) R-626, (c) G-531, and (d) G-529.

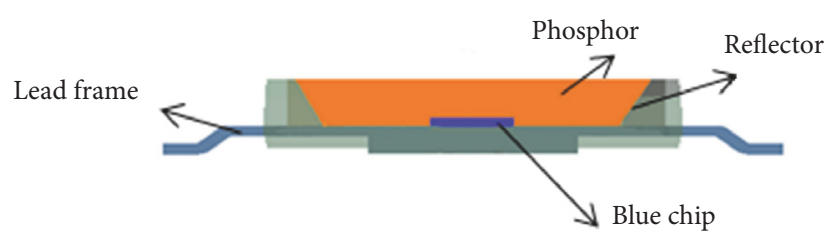

FIGURE 3: Schematic representation of the package with phosphors and a blue LED chip used in the simulation.

the empirical data measured by an integrating sphere and the red lines show the simulation data. A comparison of these curves shows that the higher the weight concentration, the higher the emission spectra intensity, although the deviations between the simulation and the empirical data were small. The CIE chromaticity coordinates of the five concentrations are listed in Table 2. The location points with values are in terms of the CIE 1931 chromaticity diagram, as shown in Figure 5. It can be seen that a weight concentration of $5 \%$ was the best choice for obtaining a magenta-colored light.

3.2. Comparison of the Blue LED Excited Radiation Power Spectrum and CIE Index for Phosphor R-626 of Different Weight Concentrations. Figure 6 shows the radiation power spectra of R-626 phosphors with weight concentrations of $0.83 \%, 1.37 \%$, and $2.11 \%$, when excited by the blue LED. The intensity of the emission spectra showed a tendency to 


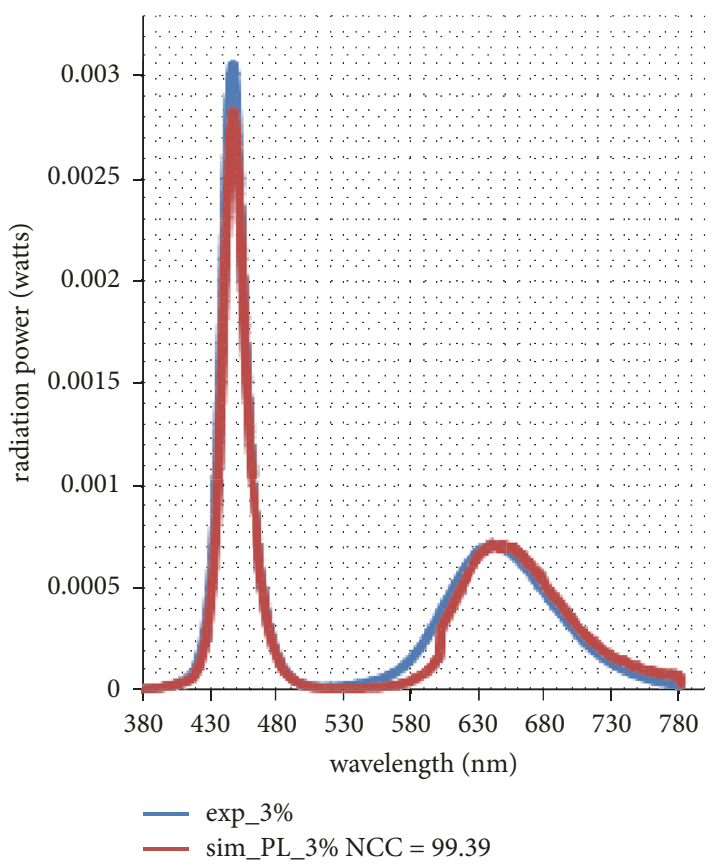

(a)

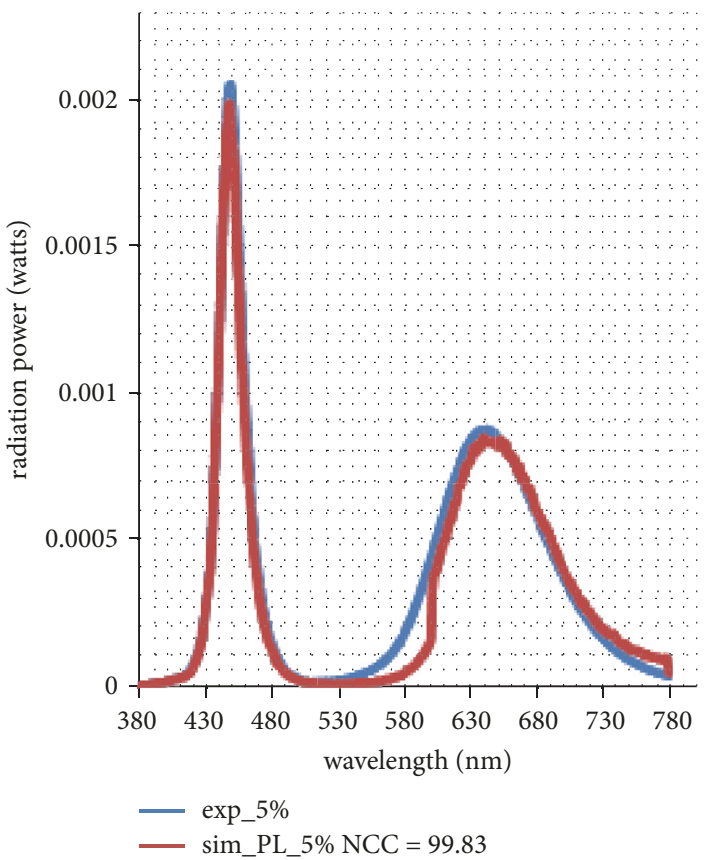

(b)

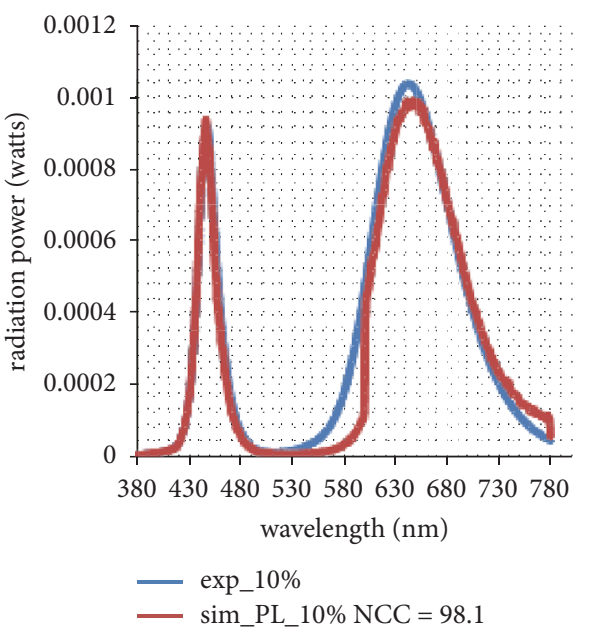

(c)

FIGURE 4: Radiation power of phosphor R-645 at three weight concentrations: (a) $3.0 \mathrm{wt} \%$; (b) $5.0 \mathrm{wt} \%$; and (c) $10.0 \mathrm{wt} \%$ with blue LED excitation.

TABLE 2: Empirical and simulated chromaticity coordinates of R-645 phosphor with three different weight concentrations with blue LED excitation.

\begin{tabular}{lcc}
\hline $\mathrm{Wt} \%$ & $\operatorname{Exp}_{-}$ & $\operatorname{Sim} \_\mathrm{PL}$ \\
& $\operatorname{CIE}(x, y)$ & $\operatorname{CIE}(x, y)$ \\
\hline $3.0 \%$ & $(0.2739,0.1065)$ & $(0.2638,0.0884)$ \\
$5.0 \%$ & $(0.3309,0.1444)$ & $(0.3138,0.1151)$ \\
$10.0 \%$ & $(0.4412,0.2114)$ & $(0.4247,0.1727)$ \\
\hline
\end{tabular}

increase as the concentration increased. The CIE coordinates are listed in Table 3. Figure 7 shows the distributions for the
TABLE 3: Empirical and simulated chromaticity coordinates of R-626 phosphor with three different weight concentrations with blue LED excitation.

\begin{tabular}{lcc}
\hline $\mathrm{Wt} \%$ & $\operatorname{Exp}_{-}$ & Sim_PL \\
& $\operatorname{CIE}(x, y)$ & $\mathrm{CIE}(x, y)$ \\
\hline $0.83 \%$ & $(0.2481,0.0946)$ & $(0.2416,0.0847)$ \\
$1.37 \%$ & $(0.2912,0.1267)$ & $(0.2801,0.1111)$ \\
$2.11 \%$ & $(0.3305,0.1568)$ & $(0.3231,0.1399)$ \\
\hline
\end{tabular}

different concentrations as illustrated in the CIE chromatic diagram. As can be seen in this figure, if magenta light 


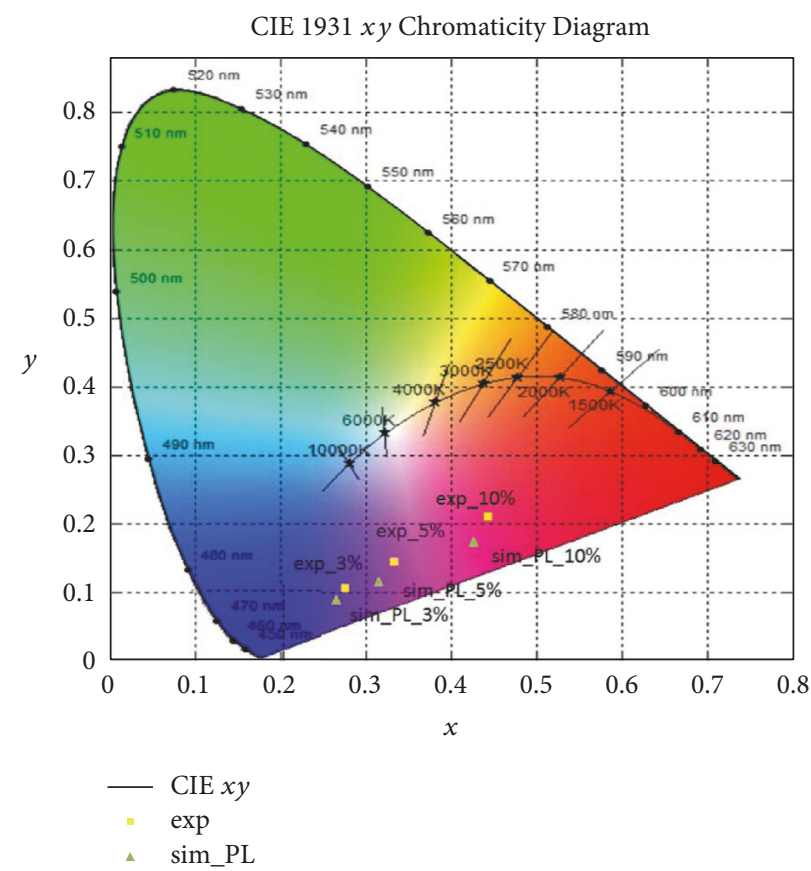

FIGURE 5: Location points on CIE 1931 chromaticity diagram for three different concentrations of phosphor R-645 excited by a blue LED.

TABLE 4: Empirical and simulated chromaticity coordinates of phosphor G-531 with three different weight concentrations under the blue LED excitation.

\begin{tabular}{lcc}
\hline $\mathrm{Wt} \%$ & $\operatorname{Exp}_{-}$ & $\operatorname{Sim\_ PL}$ \\
& $\operatorname{CIE}(x, y)$ & $\operatorname{CIE}(x, y)$ \\
\hline $5 \%$ & $(0.1988,0.2047)$ & $(0.2022,0.1857)$ \\
$10 \%$ & $(0.2158,0.2783)$ & $(0.2321,0.2792)$ \\
$20 \%$ & $(0.2460,0.3957)$ & $(0.2745,0.4017)$ \\
\hline
\end{tabular}

is required, then the $2.11 \mathrm{wt} \%$ would be a good choice for this design. Also, as discussed in Section 3.1, if light with a magenta color (R-645 phosphor at $5 \mathrm{wt} \%$ ) was required, only the $2.11 \mathrm{wt} \%$ of $\mathrm{R}-626$, for a $2.89 \mathrm{wt} \%$ reduction, would be used.

3.3. Comparison of the Radiation Power Spectrum and CIE Index of G-531 Phosphor with Different Weight Concentrations under the Blue LED Excitation. Figure 8 shows the radiation power spectra of the G-531 phosphors with weight concentrations of $5 \%, 10 \%$, and $20 \%$ under the blue LED excitation. The intensity of the emission spectra increased with the increases in the concentration. The CIE coordinates are shown in Table 4, and the distributions for the different concentrations in the CIE chromatic diagram are shown in Figure 9. As can be seen in the figure, if a design with cyanic light were demanded, then the concentration of the G-531 phosphor should be near $15 \%$.
TABLE 5: Empirical and simulated chromaticity coordinates of phosphor G-529 with three different weight concentrations under the blue LED excitation.

\begin{tabular}{lcc}
\hline $\mathrm{Wt} \%$ & Exp_ $_{-}$ & Sim_PL \\
\hline $8 \%$ & $(0.2669,0.3361)$ & CIE $(x, y)$ \\
$11.2 \%$ & $(0.2862,0.3876)$ & $(0.2804,0.3124)$ \\
$15 \%$ & $(0.3013,0.4268)$ & $(0.3101,0.3740)$ \\
\hline
\end{tabular}

3.4. Comparison of the Radiation Power Spectrum and CIE Index of G-529 Phosphors with Different Weight Concentrations under the Blue LED Excitation. Figure 10 shows the radiation power spectra of G-529 phosphors under the blue LED excitation with weight concentrations of $8 \%, 11.2 \%$, and $15 \%$. The intensity of the emission spectra also increased as the concentration increased. The CIE chromaticity coordinates are listed in Table 5, and the distributions of the different concentrations in the CIE chromatic diagram are shown in Figure 11. When cyanic light is required, the concentration of G-529 phosphor should be about $8 \%$. Compared with the results discussed in Section 3.3, where the concentration was $15 \%$, this design reduced the weight concentration by $7 \%$ and produced mixed light located at the cyan region.

\subsection{Weight Concentrations of Phosphor and Luminal Efficiency} Analysis for Four Types of Packages at a Color Temperature of $4000 \mathrm{~K}$. Figure 12 depicts the absorption and emission spectra of Y-561 phosphors and the source spectrum of the 


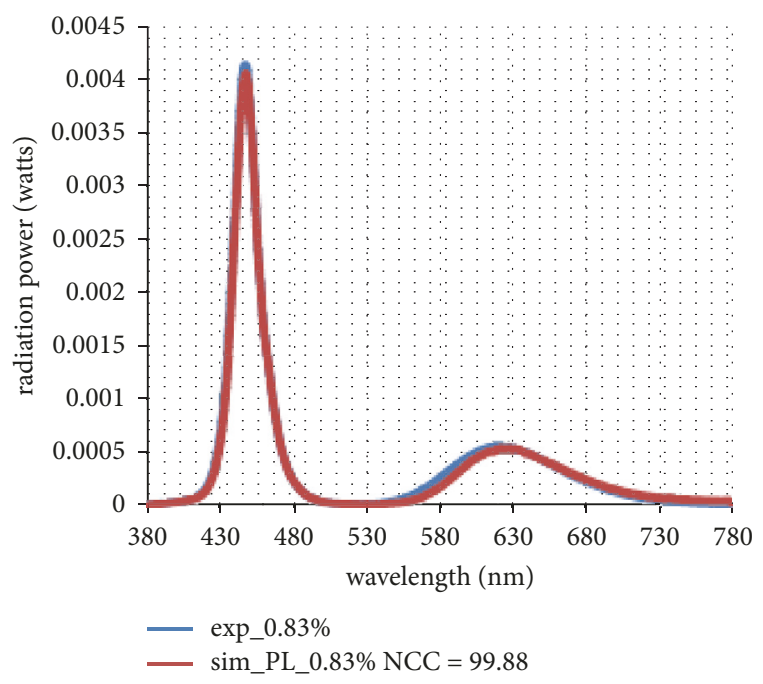

(a)

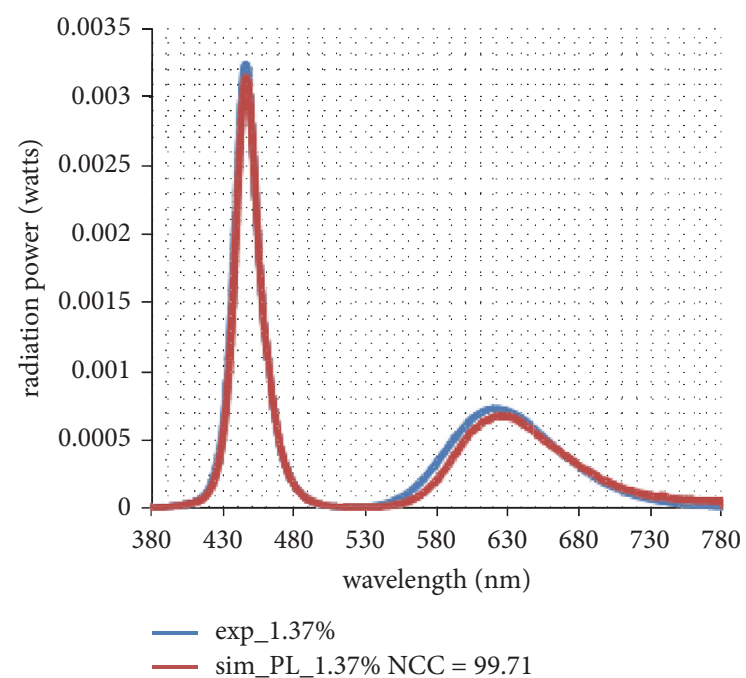

(b)

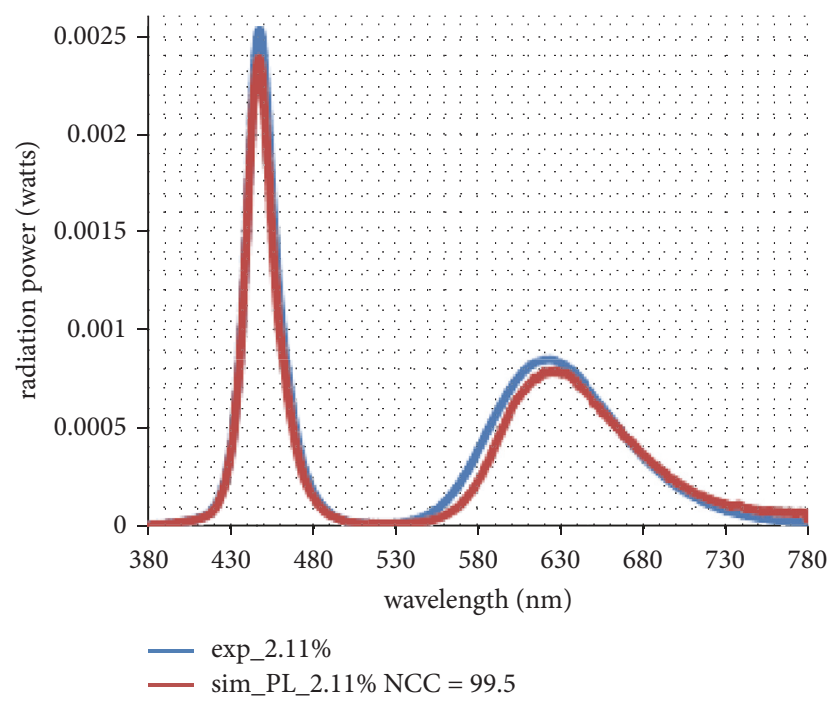

(c)

FIgURE 6: Radiation power of R-626 phosphor at three weight concentrations: (a) $0.83 \mathrm{wt} \%$; (b) $1.37 \mathrm{wt} \%$; and (c) $2.11 \mathrm{wt} \%$ with the blue LED excitation.

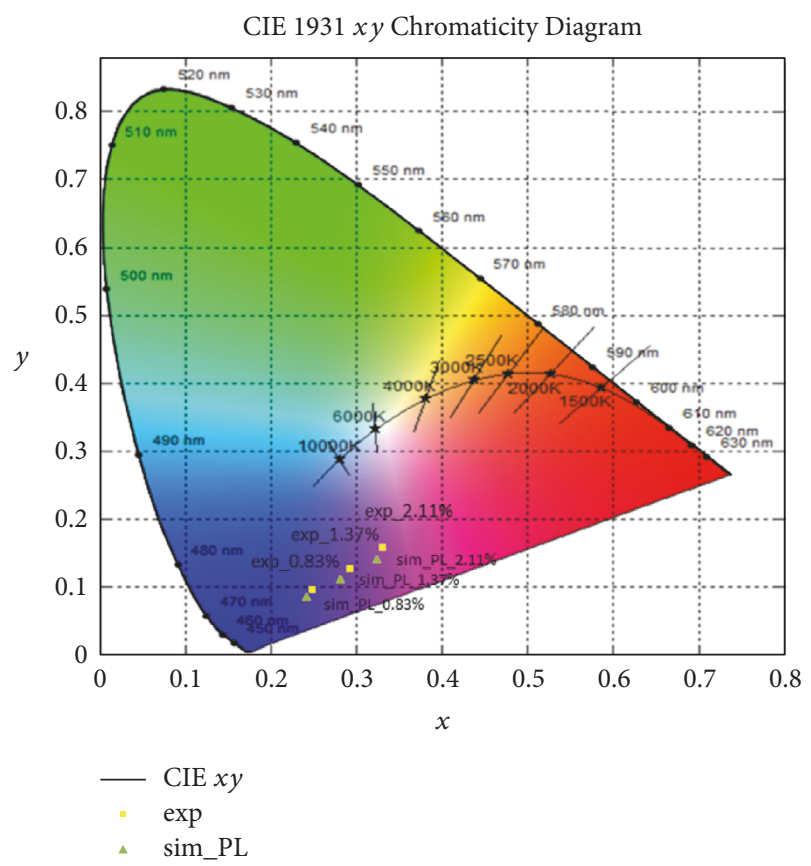

FIGURE 7: CIE chromaticity diagram from empirical and simulation data for R-626 phosphor (with three different weight concentrations) under blue LED excitation.

blue LED. Since white light can be obtained by mixing blue and yellow light, we combined a blue LED, with a spectrum peak value of $447 \mathrm{~nm}$, and adjusted the weight percentage of the Y-561 phosphor under excitation to obtain white light of the desired color temperature.

If we want the color temperature of the mixed light to be $4000 \mathrm{~K}$, we must calculate the weight concentration of Y-561 phosphor from the coordinates of the cross point of the black locus at $4000 \mathrm{~K}$ and a line connecting the location points of the blue LED and phosphor emission spectra on the CIE chromaticity diagram, as shown in Figure 13.

Figure 14 shows the four types of packages adapted in our simulation models. The weight percentages of the Y561 phosphor in these packages were manipulated to ensure that the chromaticity points were at the locus of blackbody radiation of $4000 \mathrm{~K}$ for simulation purposes; the percentages corresponding to the four types of packages, dispensing, conformal, remote, and semispherical, were $7.6 \%, 64.0 \%, 24.1 \%$, and $24 \%$, respectively. The weights of the epoxy encapsulants were $15.13 \mathrm{mg}$ (dispensing), $0.24 \mathrm{mg}$ (conformal), $6.20 \mathrm{mg}$ (remote), and $0.77 \mathrm{mg}$ (semispherical). Based on this data, the amount of phosphors used by the four types of packages could be calculated. The weights were $1.24 \mathrm{mg}$ for the dispensing type, $0.42 \mathrm{mg}$ for the conformal type, $1.97 \mathrm{mg}$ for the remote type, and $0.24 \mathrm{mg}$ for the semispherical type, as shown in Figure 15.

It was found that the Y-561 phosphor was excited by a blue LED with a power spectrum having a peak wavelength of $447 \mathrm{~nm}$ and that the semispherical type of package had a lower weight percentage than the other types for a color temperature of around $4000 \mathrm{~K}$. 


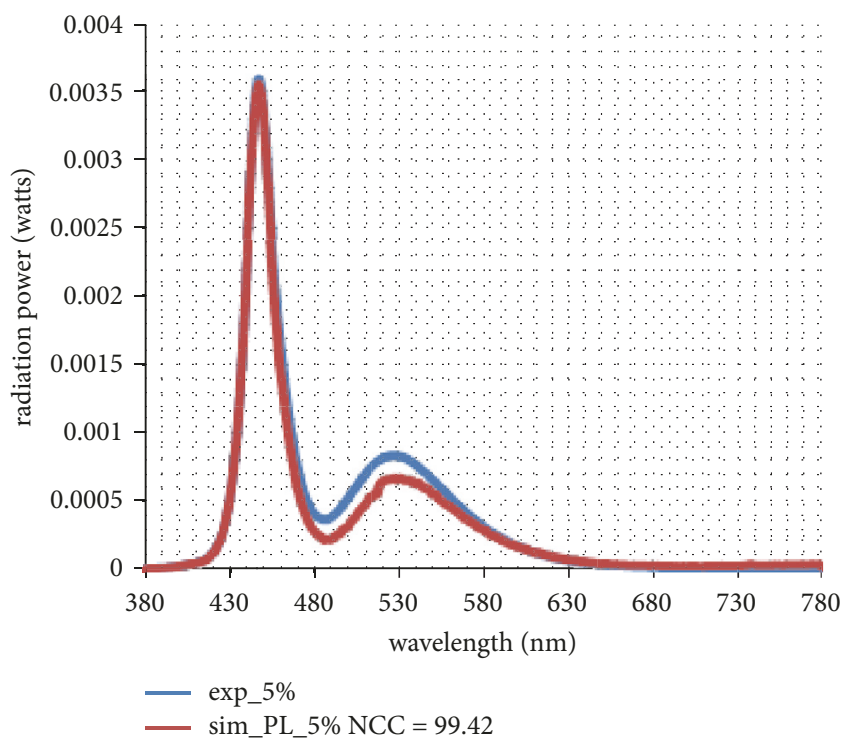

(a)

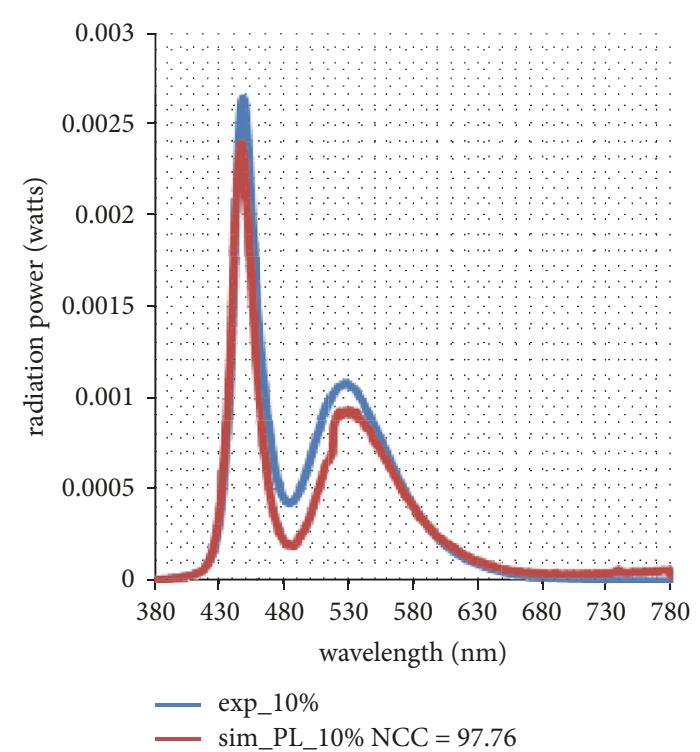

(b)

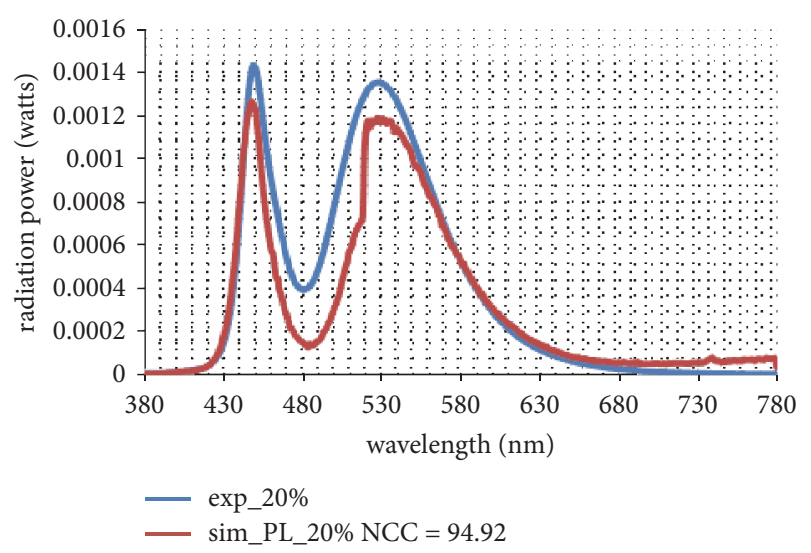

(c)

FIGURE 8: Radiation power of phosphor G-531 with three weight concentrations: (a) $5 \mathrm{wt} \%$; (b) $10 \mathrm{wt} \%$; and (c) $20 \mathrm{wt} \%$ with the blue LED excitation.

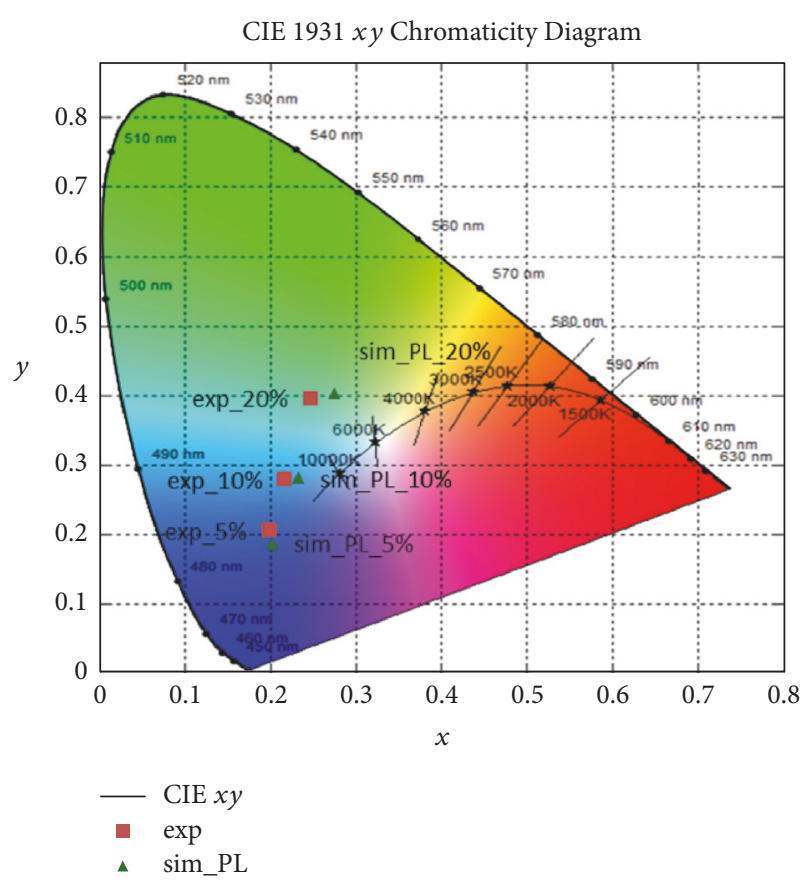

FIGURE 9: Chromaticity diagram of empirical and simulated data for G-531 phosphor with three different weight concentrations excited by a blue LED.

\section{Conclusions}

In this study, all the phosphors were excited under a blue LED with a peak power spectrum value of $447 \mathrm{~nm}$. The power of the emission spectra of the red phosphors (R645, R-626) and green phosphors (G-531, G-529) increased with an increase in the phosphor concentration. However, the deviations between the simulation and empirical curves tended to increase as the weight percentages increased, and further studies are needed to improve the results. When a 5\% weight concentration of R-645 phosphor or a $2.11 \%$ weight concentration of R-626 phosphor was combined with the blue LED, chromaticity coordinates near the magenta area were obtained. When an $8 \%$ weight concentration of G-529 phosphor was combined with a blue LED, the mixed light was within the cyan spectrum.

To produce a mixed white light with a color temperature of around $4000 \mathrm{~K}$ with phosphor Y-561 and the blue LED, the concentration of phosphors was calculated from the intersection coordinates of the two lines on the CIE chromaticity diagram: the first being the locus of blackbody radiation at $4000 \mathrm{~K}$; the other line being the connection points between the blue LED spectrum and the Y-561 phosphor emission spectrum. The chromaticity coordinates of this point were $x=0.3804$ and $y=0.3768$. The simulations for the packages revealed that the semispherical type used the least amount of Y-561 phosphor to achieve a color temperature of $4000 \mathrm{~K}$. 


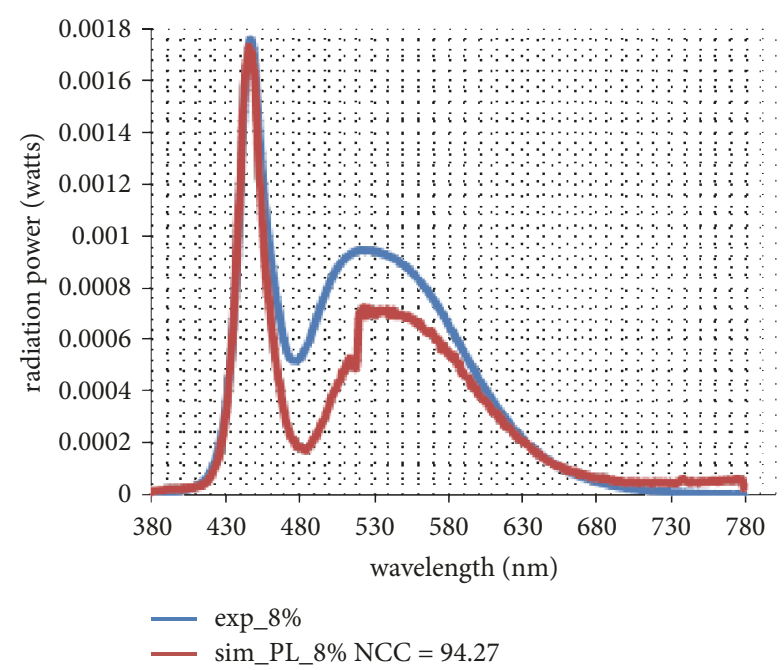

(a)

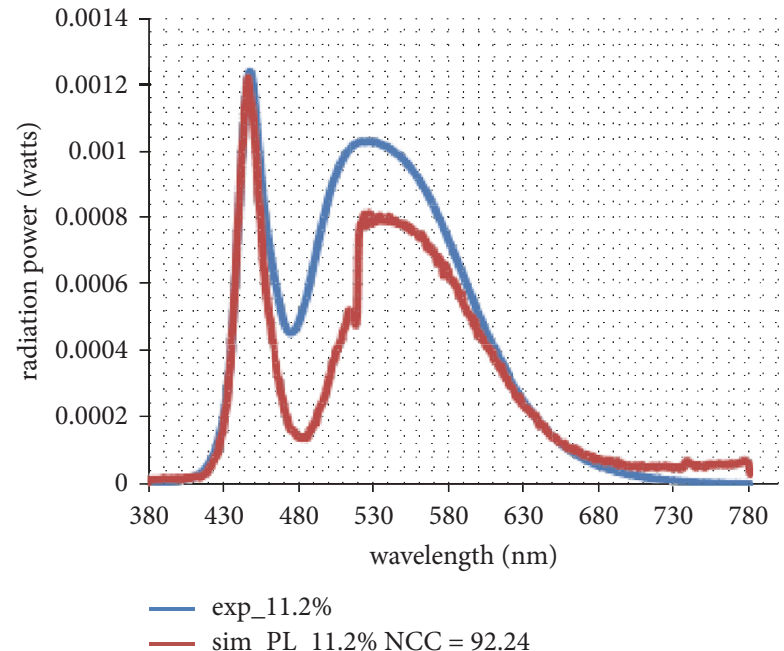

(b)

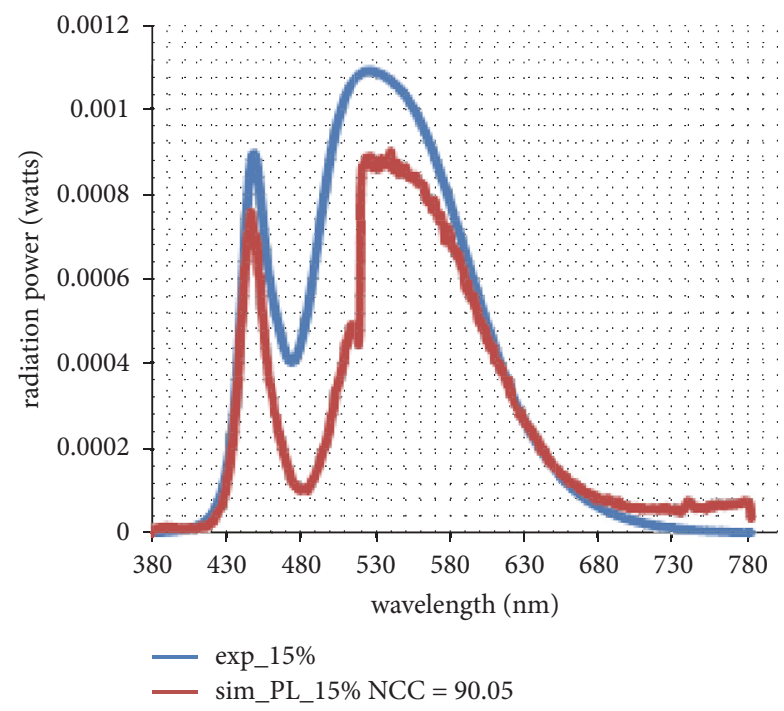

(c)

FIGURE 10: Radiation power of G-529 phosphor with three weight concentrations: (a) $8 \mathrm{wt} \%$; (b) $11.2 \mathrm{wt} \%$; and (c) $15 \mathrm{wt} \%$ excited by the blue LED.

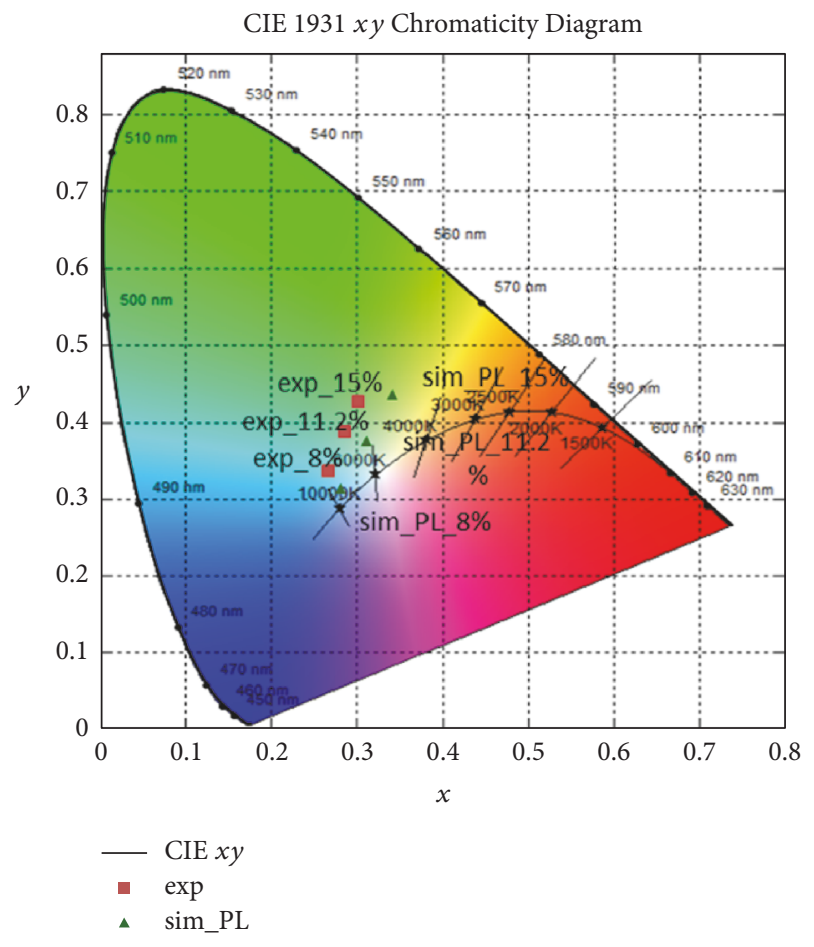

FIGURE 11: Chromaticity diagram of G-529 phosphors with three different weight concentrations excited by a blue LED.

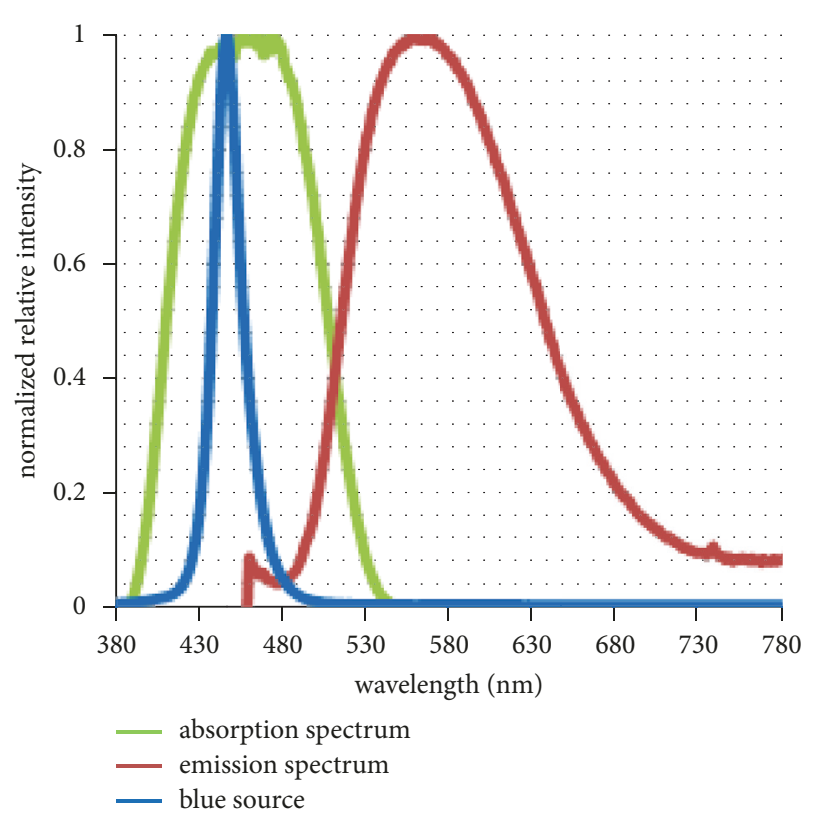

FIgURE 12: Power spectrum of the blue LED and the emission and absorption spectra of Y-561 phosphor.

\section{Conflicts of Interest}

The authors declare that there are no conflicts of interest regarding the publication of this paper. 


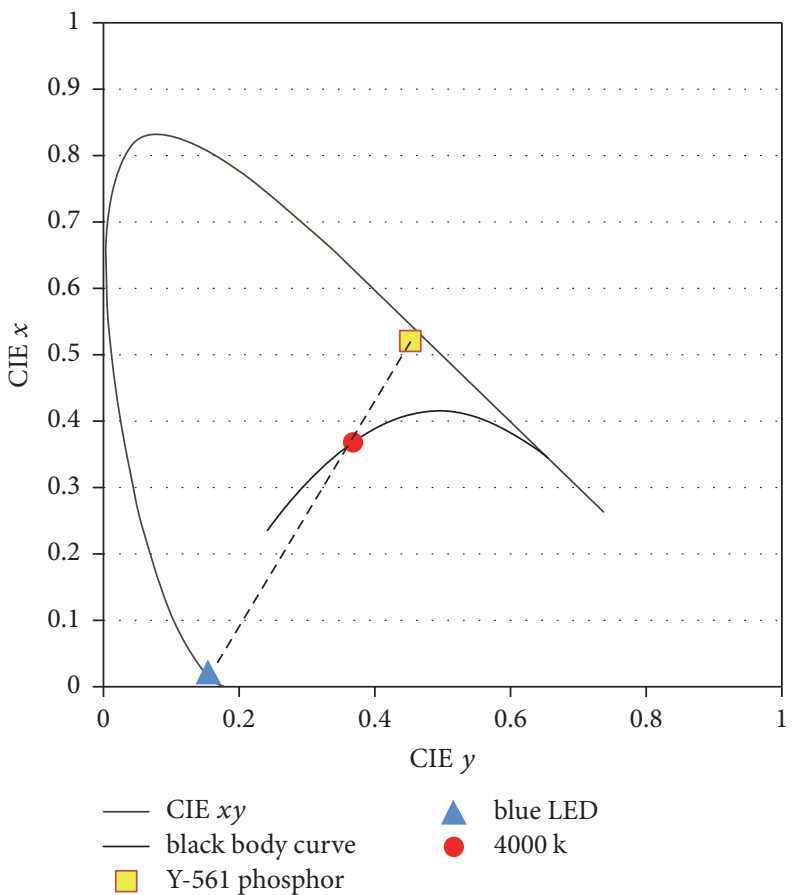

FIGURE 13: Schematic for location determination of white light from the points in the CIE chromaticity diagram of the blue LED and yellow Y-561 phosphor.

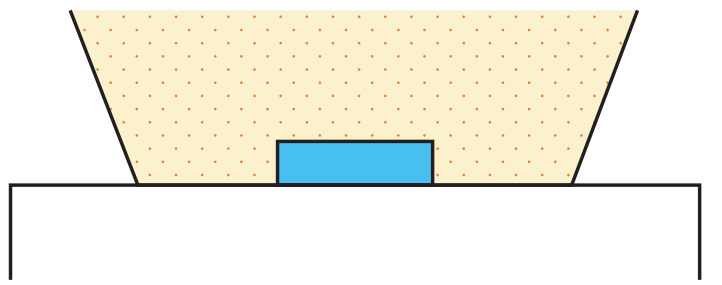

(a)

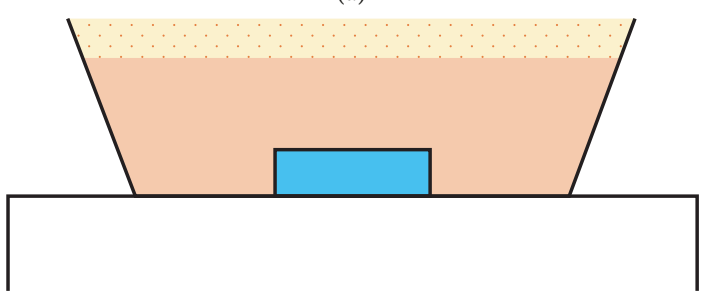

(c)

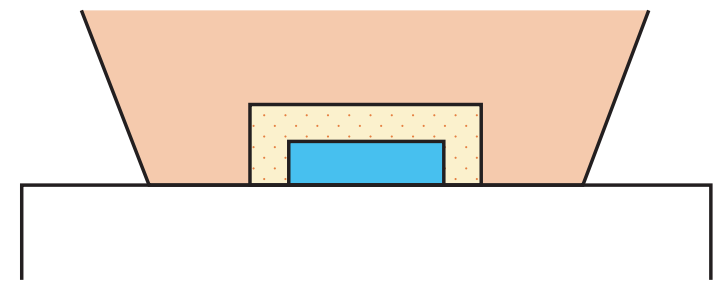

(b)

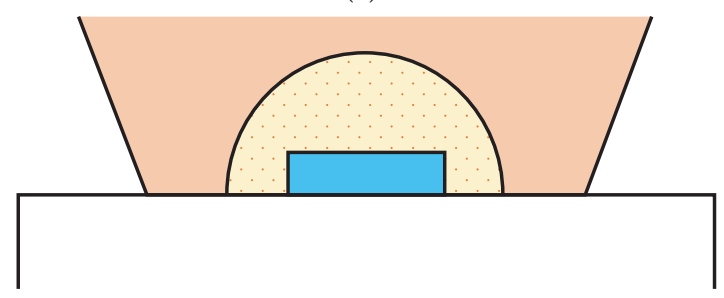

(d)

FIGURE 14: Four types of packages for simulation: (a) dispensing; (b) conformal; (c) remote; (d) semispherical.

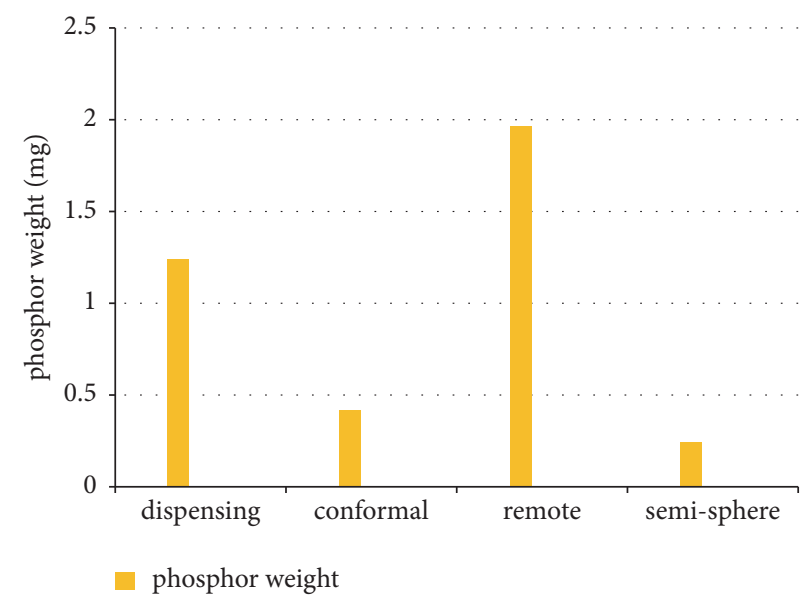

FIGURE 15: Comparison of light efficiency with phosphor weight for the four package types. 


\section{Acknowledgments}

This study was sponsored by the Ministry of Science and Technology, Taiwan, under Contracts nos. MOST106-2221-E008-075 and MOST 106-2221-E-035-072-MY2.

\section{References}

[1] S. Pimputkar, J. S. Speck, S. P. Denbaars, and S. Nakamura, "Prospects for LED lighting," Nature Photonics, vol. 3, no. 4, pp. 180-182, 2009.

[2] Y. Shimizu, K. Sakano, Y. Noguchi, and T. Moriguchi, U.S. patent 5998925, 1999.

[3] A. A. Setlur, A. M. Srivastava, H. A. Comanzo, and D. D. Doxsee, U.S. patent 6685852, 2004.

[4] Y. H. Won, H. S. Jang, K. W. Cho, Y. S. Song, D. Y. Jeon, and H. K. Kwon, "Effect of phosphor geometry on the luminous efficiency of high-power white light-emitting diodes with excellent color rendering property," Optics Letters, vol. 34, no. 1, pp. 1-3, 2009.

[5] C.-Y. Chen, T.-H. Yang, C.-H. Hsu, and C.-C. Sun, "Highefficiency white LED packaging with reduced phosphor concentration," IEEE Photonics Technology Letters, vol. 25, no. 7, pp. 694-696, 2013.

[6] T.-H. Yang, C.-Y. Chen, Y.-Y. Chang et al., "Precise Simulation of Spectrum for Green Emitting Phosphors Pumped by a Blue LED Die," IEEE Photonics Journal, vol. 6, no. 4, 2014.

[7] M.-T. Wang and J.-M. Huang, "Accurate control of chromaticity and spectra by feedback phosphor-coating," Optics Express, vol. 23, no. 9, pp. 11576-11585, 2015.

[8] S. A. Schafer, "Quasi-Monte Carlo methods: applications to modeling of light transport in tissue," in Proceedings of the LaserTissue Interaction VII, vol. 2681, pp. 317-324, SPIE Proceedings, 1996.

[9] S. J. Lee, "Analysis of light-emitting diodes by Monte Carlo photon simulation," Applied Optics, vol. 40, no. 9, pp. 1427-1437, 2001.

[10] G. Mie, "Beiträge zur Optik trüber Medien, speziell kolloidaler Metallösungen," Annalen der Physik, vol. 330, no. 3, pp. 377-445, 1908.

[11] C.-C. Sun, T.-X. Lee, S.-H. Ma, Y.-L. Lee, and S.-M. Huang, "Precise optical modeling for LED lighting verified by cross correlation in the midfield region," Optics Expresss, vol. 31, no. 14, pp. 2193-2195, 2006.

[12] J. Fox, Applied Regression Analysis, Linear Models, and Related Methods, SAGE Publications, Incorporated, 1997. 

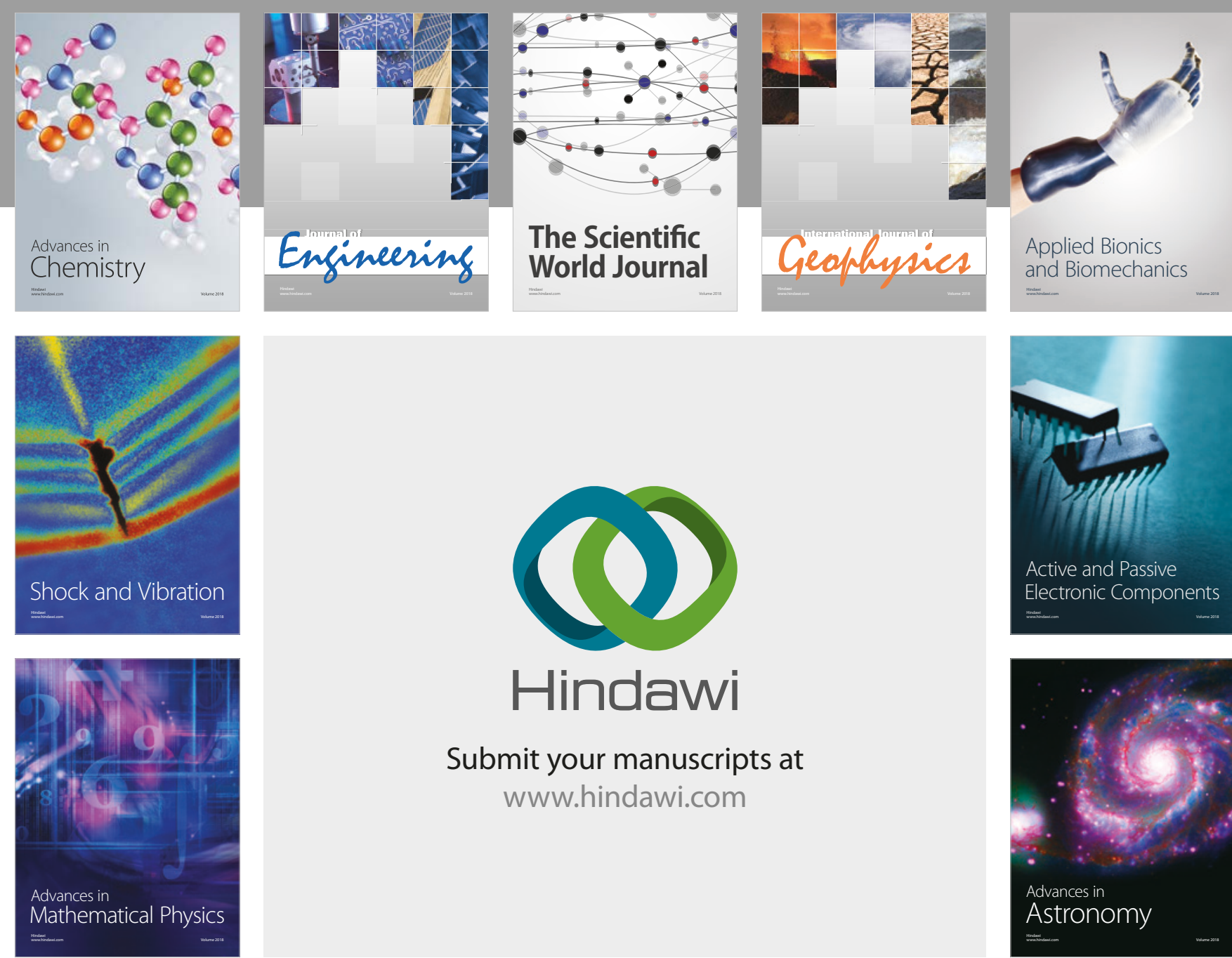

Submit your manuscripts at

www.hindawi.com

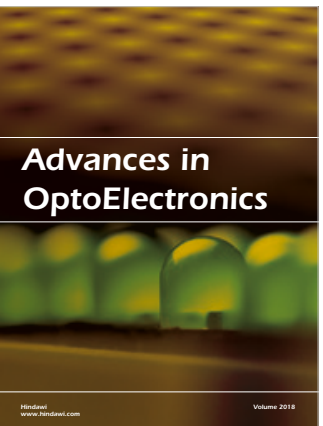

\section{Rotcting Machinery}
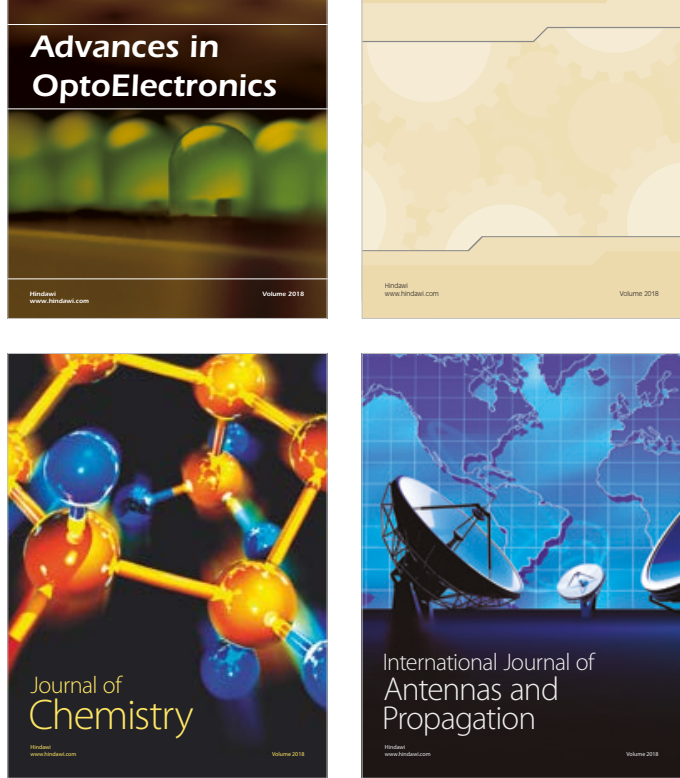

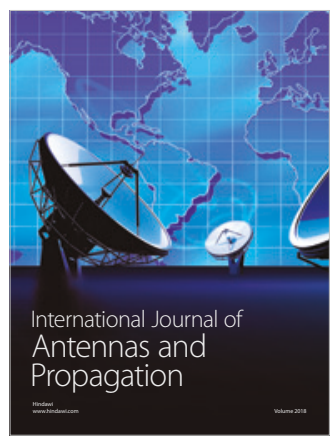

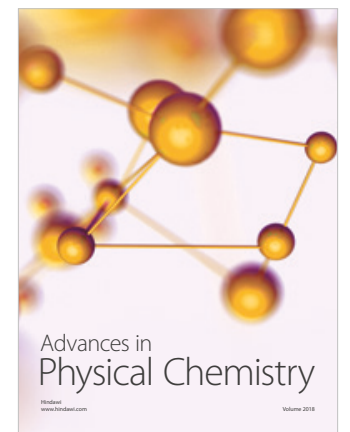

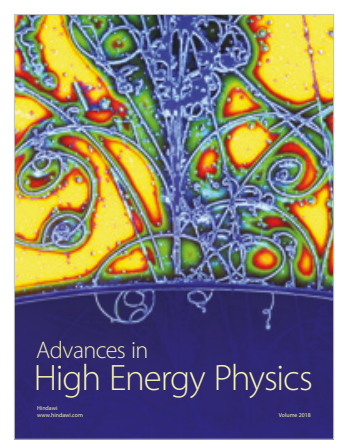

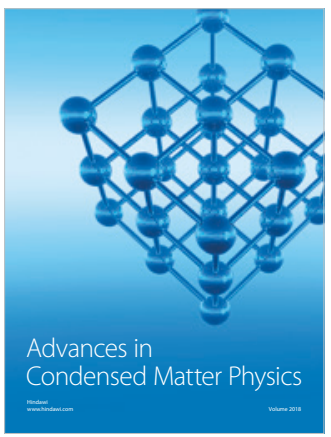

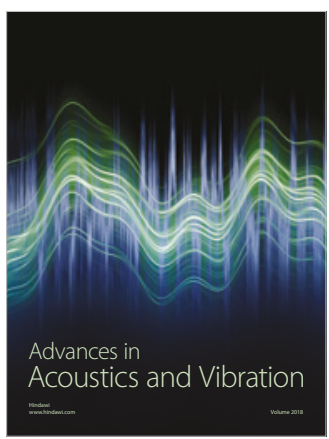

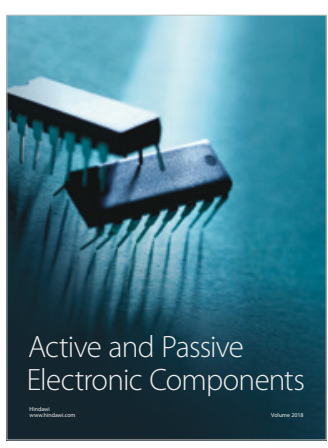
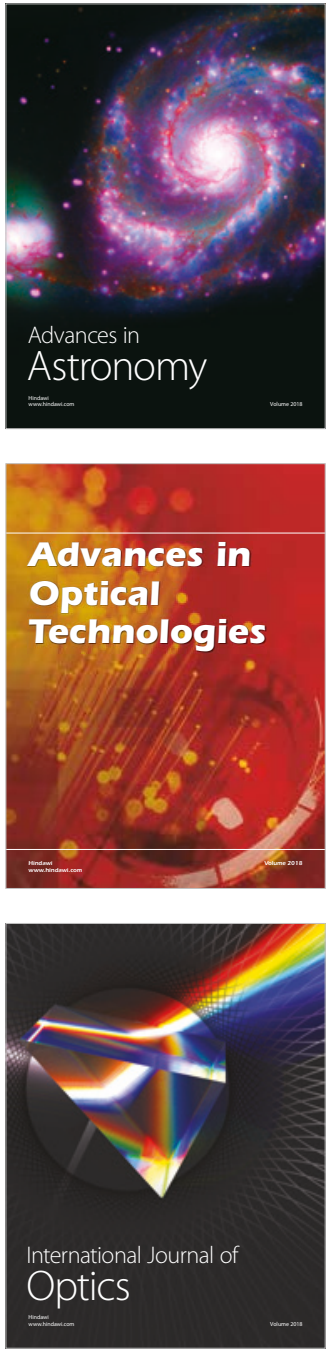